\title{
MELHORAMENTO DO FEIJOEIRO COMUM VISANDO A OBTENÇÃO DE CULTIVARES PRECOCES COM GRÃOS TIPO 'CARIOCA' E 'ROŞINHA'
}

\author{
Common bean breeding for obtaining early cultivars with 'carioca' or 'rosinha' grain type
}

\author{
Aisy Botega Baldoni'1, João Bosco dos Santos², Ângela de Fátima Barbosa Abreu ${ }^{3}$
}

\begin{abstract}
RESUMO
Visando associar na seleção de novos genótipos de feijoeiro comum características favoráveis como o tipo de grão, a produção de grãos e a resistência à mancha angular e antracnose, foi realizado o cruzamento entre a cultivar Rosinha e a linhagem ESAL 693, ambas precoces e com hábito de crescimento tipo I. Adicionalmente, a linhagem ESAL 693 é resistente à mancha angular e à antracnose e a 'Rosinha' é suscetível a ambas. As famílias foram avaliadas da geração $\mathrm{F}_{2: 3}$ até $\mathrm{F}_{2: 6}$ sendo ao final, selecionadas as 23 superiores. Em todos os experimentos foi utilizado o delineamento látice e como testemunhas a ESAL 693 e 'Rosinha'. Foi observada ampla variabilidade genética entre as famílias incluindo as 23 selecionadas, tendo sido obtidas estimativas de herdabilidade de $51,29 \%$ para a produtividade de grãos, 74,11\% para o tipo de grão e 93,64\% para a reação à mancha angular. Nove famílias destacaram-se em relação às três características estudadas; porém, duas com grãos semelhantes ao 'Carioca' e uma com grão semelhante ao 'Rosinha', apresentando também resistência à antracnose e boa capacidade de cozimento. Existe a possibilidade de seleção, dentro dessas famílias, de linhagens precoces e que reúnam as características desejadas.
\end{abstract}

Termos para indexação: Phaseolus vulgaris, famílias segregantes, Phaeoisariopsis griseola, seleção, Colletotrichum lindemuthianum.

\begin{abstract}
Aiming to select common bean plants with high grain yield, ideal grain type and resistant to anthracnose and angular leaf spot, the cultivar Rosinha and the line ESAL 693 were crossed. Both have early cycle, growth habit I, and ideal grain types. ESAL 693 is resistant to angular leaf spot and anthracnose, and 'Rosinha' is susceptible for both traits. The families were evaluated from F- ${ }_{2: 3}$ to $\mathrm{F}_{2: 6}$ generation, and the best 23 were selected. Square lattice design, and the checks 'Rosinha' and ESAL 693 were used in all experiments except in the first when 'Rosinha' was not included. A broad genetic variability was detected even among the remaining 23 families, when the heritability estimated for grain yield was $51,29 \%$, for grain type was $74,11 \%$, and for resistance to angular leaf spot was $93,64 \%$. Among the best nine families in these three traits, two with 'Carioca' grain type and one with 'Rosinha' were also resistant to anthracnose and higher cooking capacity. So, there is a possibility to select lines with the desired phenotypes within these families.
\end{abstract}

Index terms: Phaseolus vulgaris, segregating families, Phaeoisariopsis griseola, selection, Colletotrichum lindemuthianum.

(Recebido para publicação em 3 de janeiro de 2005 e aprovado em 22 de abril de 2005)

\section{INTRODUÇÃO}

A possibilidade de cultivar o feijoeiro comum no período de outono/inverno, com o uso da irrigação, em regiões de inverno ameno, despertou o interesse de grandes agricultores de algumas regiões de Minas Gerais. A expansão da cultura nesse período vem aumentando significativamente e o uso de cultivares precoces proporcionam vantagens como, por exemplo, economia na irrigação.

Entretanto, nessas condições de cultivo a ocorrência de doenças como a antracnose (Colletotrichum lindemuthianum) e mancha angular (Phaeoisariopsis griseola), têm causado significativas reduções de produção no Sul de Minas Gerais. No caso das cultivares do grupo Roxo e Rosinha, a ferrugem (Uromyces appediculatus) é também um sério problema, porque em geral elas são altamente suscetíveis (POMPEU, 1993; SARTORATO \& RAVA, 1994). Assim, uma medida de controle ideal seria a obtenção de novas cultivares com resistência aos patótipos mais freqüentes na região.

Além da resistência aos principais patógenos, outras características importantes são a produtividade e o tipo de grãos. Este último é um dos fatores determinantes para a aceitação do produto no mercado (PEREIRA et al., 2004). Além do tipo semelhante ao da cultivar Carioca, o tipo 'Rosinha' também é aceito em algumas regiões (RAMALHO \& ABREU, 1998).

Considerando esses aspectos objetivou-se com o presente trabalho avaliar e selecionar famílias provenientes

\footnotetext{
${ }^{1}$ Mestranda em Agronomia/Genética e Melhoramento de Plantas, bolsista do CNPq, Universidade Federal de Lavras/UFLA - Cx. P. 3037 - $37200-000$ Lavras, MG

2Professor titular do DBI, Universidade Federal de Lavras/UFLA - Cx. P. 3037 - 37200-000 - Lavras, MG - jbsantos@ufla.br

${ }^{3}$ Pesquisadora da EMBRAPA Arroz e Feijão, DBI, Universidade Federal de Lavras/UFLA - Cx. P. 3037 - 37200-000 - Lavras, MG.
} 
do cruzamento ESAL 693 x 'Rosinha', em dois locais do Sul de Minas Gerais, com base na produtividade de grãos, resistência à mancha angular e à antracnose e que possuam tipos de grãos semelhantes às cultivares carioca e rosinha.

\section{MATERIAL E MÉTODOS}

Foram utilizadas 47 famílias segregantes provenientes do cruzamento entre a cultivar Rosinha e a linhagem ESAL 693. A 'Rosinha' pertence ao grupo Rosinha, é suscetível aos agentes causais da mancha angular e da antracnose apresenta grão pequeno e baixa produtividade, grão de cor creme clara e halo rosa com aceitação comercial em algumas regiões brasileiras; a ESAL 693 pertence ao grupo Carioca com tipo de grão semelhante ao da cultivar Carioca com listras levemente escuras, é resistente aos agentes causais da mancha angular e da antracnose (alelo Co-5) e apresenta alto potencial produtivo. Ambas são precoces e com hábito de crescimento tipo I.

Do cruzamento foi obtida a geração $F_{1}$ que gerou 120 plantas $\mathrm{F}_{2}$. As sementes colhidas de cada planta constituíram uma família $\mathrm{F}_{2: 3}$. Essas famílias e o genitor ESAL 693 foram avaliados em um experimento no delineamento látice simples 11 x 11, no município de Lavras na safra da 'seca' de 2001 (semeadura em fevereiro). Cada parcela foi composta por uma linha de um metro com 15 sementes. A adubação foi realizada com $300 \mathrm{~kg} / \mathrm{ha}$ da fórmula 8-28-16. Cerca de 20 dias após a emergência foi realizada adubação nitrogenada de cobertura à base de 20 $\mathrm{kg} / \mathrm{ha}$ de nitrogênio. Durante a condução do experimento a irrigação foi realizada periodicamente, sempre que necessária. Setenta e nove famílias $\mathrm{F}_{2: 4}$, selecionadas com base principalmente no tipo de grãos semelhantes ao 'Carioca' e 'Rosinha', foram novamente avaliadas no inverno de 2001 (semeadura em julho), juntamente com os genitores, no município de Ijaci, em parcelas de 2 linhas de 1 metro. Foram adotados procedimentos experimentais semelhantes aos da seca de 2001. Novamente procedeuse a seleção das famílias, principalmente quanto à produção e tipo de grãos e foram selecionadas as $47\left(\mathrm{~F}_{2.5}\right)$ superiores. Essas famílias, juntamente com os genitores, foram avaliadas em um látice triplo $7 \times 7$, com parcelas de duas linhas de 2 m, na safra da 'seca' de 2002, nos municípios de Lavras e Lambari. Foram selecionadas as 23 famílias superiores $\left(\mathrm{F}_{2: 6}\right)$ e, juntamente com os genitores, foram avaliadas no inverno de 2002 no município de Ijaci em um látice triplo $5 \times 5$, com parcelas de duas linhas de $2 \mathrm{~m}$. Essas famílias foram também inoculadas com a raça 73 de Colletotrichum lindemuthianum, o agente causal da antracnose, utilizando a metodologia descrita por Silva \& Santos (2001).

$\mathrm{Na}$ avaliação das famílias foram considerados a produção, o tipo de grão e a resistência à mancha angular. O tipo de grão foi avaliado com base em uma escala descritiva de notas, de acordo com a proposta de Marques Júnior (1997), sendo 1 o tipo mais aceito pelo mercado ('Carioca' ou 'Rosinha') e 5 o mais diferente e inaceitável pelo mercado consumidor. A severidade da mancha angular foi avaliada com base em um diagrama de notas de 1 (resistente) a 9 (suscetível), segundo Sartorato (2001).

A produção de grãos foi avaliada em todos os experimentos, exceto na 'seca' de 2001 em Lavras, devido a problemas experimentais. $\mathrm{O}$ tipo de grãos foi avaliado experimentalmente apenas em $\mathrm{F}_{2: 5}$ (Lambari) e em $\mathrm{F}_{2: 6}$ (Ijaci). Entretanto, especialmente $\mathrm{em}_{2}$, bem como nas demais gerações segregantes, foram eliminadas as sementes desfavoráveis (seleção massal). A reação à mancha angular foi avaliada nos experimentos conduzidos na safra da 'seca' em $F_{2: 5}$ (Lavras e Lambari), quando as condições ambientais favorecem a incidência do patógeno.

Foram realizadas análises de variâncias individuais e conjuntas para a produtividade e tipo de grãos e reação à mancha angular, considerando o efeito de famílias e ambientes como fixos. Foram estimados também os coeficientes de determinação genotípicos, equivalentes das herdabilidades no sentido amplo (VENCOVSKY \& BARRIGA, 1992).

\section{RESULTADOS E DISCUSSÃO}

A partir das avaliações das famílias $\mathrm{F}_{2: 3}$ foram selecionadas as mais promissoras considerando-se o tipo de grãos nas primeiras gerações segregantes, a reação à mancha angular nas avaliações da safra da 'seca', que é a ideal para a ocorrência da doença (PAULA JÚNIOR \& ZAMBOLIM, 1998; SARTORATO \& RAVA 1994), e também a produção de grãos, especialmente nas últimas avaliações. Conseqüentemente, várias famílias foram eliminadas até a última avaliação, na geração $\mathrm{F}_{2: 6}$ Por isso, são apresentados apenas os resultados das análises conjuntas envolvendo as 23 famílias selecionadas no caso da produtividade e tipo de grãos e das 48 para a reação à mancha angular.

Observa-se na Tabela 1 que ainda há ampla variabilidade genética entre as 23 famílias selecionadas com base na produção e tipo de grãos. Nota-se, também, uma interação altamente significativa de famílias por ambientes, quando se considera a produção e a reação à mancha angular. No caso de produção a interação é comumente observada

Ciênc. agrotec., Lavras, v. 30, n. 1, p. 67-71, jan./fev., 2006 
(RAMALHO et al., 1993). Em relação à mancha angular, a interação sugere a presença de diferentes patótipos do patógeno nos dois locais, uma vez que tem sido relatada grande variabilidade do fungo no Estado de Minas Gerais e a composição racial geralmente é particular para cada região (MENDONÇA et al., 2003; NIETSCHE et al., 2001; SARTORATO \& RAVA, 1994). Em relação ao tipo de grãos a seleção é mais fácil por não ter sido detectada interação genótipos x ambientes. Refletindo a ampla variabilidade genética entre as famílias, os valores de herdabilidade foram $51,29 \%$ para a produtividade de grãos, $74,11 \%$ para o tipo de grãos e $93,64 \%$ para a reação a mancha angular.

Como as famílias são segregantes havendo a possibilidade de seleção de linhagens dentro das mesmas, inicialmente, a estratégia mais adequada é a seleção daquelas superiores para as três características estudadas. Assim, observa-se na Tabela 2 o valor médio dessas características a partir de todas as avaliações realizadas.

Em relação à produção de grãos os genitores não diferiram, embora, ao contrário do esperado, a cultivar Rosinha tenha mostrado tendência de ser superior à ESAL 693. Essa superioridade ocorreu principalmente na avaliação de inverno, quando as condições ambientais foram as melhores, propiciando as maiores produtividades médias de grãos. No entanto, com o presente estudo, foi possível identificar algumas famílias superiores a ambos genitores. Considerando que as famílias são segregantes, há a possibilidade de seleção de linhagens com produtividades superiores à média das melhores famílias.

Todas as famílias selecionadas foram superiores à ESAL 693 quanto ao tipo de grãos e semelhante a 'Rosinha', como esperado. A cor de fundo e as listras levemente escuras, indesejáveis do genitor ESAL 693 foram eliminadas durante as seleções. Em relação a esse caráter não há restrição para a seleção de linhagens superiores em qualquer das 23 famílias segregantes.

Quatorze famílias foram resistentes à mancha angular, devido ao genitor ESAL 693 apresentar um alelo dominante de resistência a esta enfermidade (VIEIRA, 2004). Não foi observada a ocorrência da antracnose nos experimentos de campo, embora eles tenham sido realizados juntamente com outros experimentos, nos quais a doença foi observada em algumas cultivares suscetíveis. O genitor ESAL 693 é também portador do alelo Co-5, que confere resistência à maioria das raças de $C$. lindemuthianum que ocorrem no Brasil (RAVA et al., 1994); portanto, há também a possibilidade das famílias selecionadas apresentarem esse alelo.

É importante salientar que nove famílias destacaram-se em relação às três características estudadas, sendo cinco $(26,27,58,69 \mathrm{C}$ e 80$)$ com tipo de grãos semelhantes ao da cultivar carioca, e quatro $(3,37,55$ e 75$)$ semelhantes ao da 'Rosinha' (Tabela 2). Entre estas, a 3, $27,55,58$ e 75 apresentaram maior resistência à ferrugem no inverno de 2002, com destaque especial para as famílias com sementes semelhantes ao 'Rosinha', uma vez que as cultivares deste grupo são tradicionalmente muito suscetíveis a essa doença (POMPEU, 1993). Entretanto, a ferrugem não tem causado danos significativos na região Sul de Minas Gerais, provavelmente porque as condições ambientais não são as mais favoráveis para a doença (PAULA JÚNIOR \& ZAMBOLIM, 1998).

TABELA 1 - Resumo das análises conjuntas de variância da produção, tipo de grão e reação à mancha angular, com as respectivas estimativas de alguns parâmetros genéticos, relativos às avaliações de famílias selecionadas no cruzamento ESAL 693 x 'Rosinha'.

\begin{tabular}{lcccccc}
\hline \multirow{2}{*}{ Fontes de Variação } & \multicolumn{2}{c}{ Produção grãos(g/parcela) } & \multicolumn{2}{c}{ Tipo de grãos $^{\mathbf{1}}$} & \multicolumn{2}{c}{ Reação a $P$. griseola $^{\mathbf{1}}$} \\
\cline { 2 - 7 } & GL & QM x10 & GL & QM & GL & QM \\
\hline Ambientes(A) & 3 & $1785,01^{* *}$ & 1 & $0,628^{* *}$ & 1 & $3,813^{* *}$ \\
Famílias(F) & 24 & $4,37^{* *}$ & 24 & $0,197 * *$ & 48 & $4,308^{* *}$ \\
A x F & 72 & $3,91^{* *}$ & 24 & 0,071 & 48 & $0,769 * *$ \\
Erro médio & 128 & 2,13 & 72 & 0,051 & 156 & 0,274 \\
\hline CV $(\%)$ & & 9,36 & 10,29 & & 12,83 \\
$\mathrm{~h}_{\mathrm{a}}{ }^{2}(\%)$ & 51,29 & & 74,11 & & 93,64 \\
\hline
\end{tabular}

${ }^{1}$ Notas de 1 (mais aceito pelo consumidor) a 5 (menos aceito pelo consumidor) para tipo de grãos e 1 (mais resistente) a 9 (mais suscetível) para reação a $P$. griseola.

** Significativo a $1 \%$ de probabilidade pelo teste $\mathrm{F}$. 
Um dos objetivos do uso do genitor ESAL 693 foi a introdução do alelo de resistência à antracnose Co-5, que confere resistência à maioria dos patótipos de $C$. lindemuthianum prevalecentes no Brasil (RAVA et al., 1994). Entre as famílias mais promissoras a 26, 69C, 37 e 55 mostraram-se segregantes quando inoculadas com a raça 73 , indicando ser possível a seleção de linhagens resistentes.

Foi constatado que ESAL 693 tem baixa capacidade de cozimento dos grãos e o genitor 'Rosinha' tem boa capacidade de cozimento (BALDONI \& SANTOS, 2005).
Entre as famílias mais promissoras, selecionadas com base nas outras características avaliadas, as famílias 26, 29C e 37 possuem capacidade de cozimento semelhante ao genitor 'Rosinha', além de terem exibido variação genética dentro, indicando a possibilidade de seleção de linhagens em relação a esse caráter. A família 55, embora favorável em relação aos outros caracteres não se destacou em relação à capacidade de cozimento. Portanto, sobressaíram as famílias 26 e 29C com sementes tipo 'Carioca' e a família 37, com sementes tipo 'Rosinha', dentro das quais poderão ser selecionadas linhagens promissoras.

TABELA 2 - Médias das 23 melhores famílias selecionadas no cruzamento ESAL 693 x 'Rosinha' relativas à produção, tipo de grão e reação a Phaeoisariopsis griseola.

\begin{tabular}{|c|c|c|c|c|}
\hline \multirow{2}{*}{ Famílias } & \multirow{2}{*}{$\begin{array}{l}\text { Produção grãos } \\
\text { (g/parcela) }\end{array}$} & \multicolumn{2}{|c|}{ Tipo grão } & \multirow{2}{*}{ Reação $P$. griseola ${ }^{I}$} \\
\hline & & Cor do grão & Nota $^{1}$ & \\
\hline 26 & $536,5 \mathrm{a}^{2}$ & Carioca & $1,77^{1} \mathrm{a}^{2}$ & $3,17^{1} \mathrm{a}^{2}$ \\
\hline $72 \mathrm{R}$ & $481,8 \mathrm{~b}$ & Rosinha & $2,25 \mathrm{a}$ & $2,32 \mathrm{a}$ \\
\hline 47 & 528,4 a & Rosinha & $2,18 \mathrm{a}$ & $4,96 \mathrm{c}$ \\
\hline 55 & 514,9 a & Rosinha & $2,11 \mathrm{a}$ & $2,32 \mathrm{a}$ \\
\hline 64 & $460,5 \mathrm{~b}$ & Rosinha & 2,11 a & 3,33 a \\
\hline 4 & $452,3 \mathrm{~b}$ & Rosinha & $1,87 \mathrm{a}$ & 3,41 a \\
\hline $8 \mathrm{R}$ & $492,9 \mathrm{a}$ & Rosinha & $1,99 \mathrm{a}$ & $4,80 \mathrm{c}$ \\
\hline 75 & 549,0 a & Rosinha & $2,00 \mathrm{a}$ & $2,90 \mathrm{a}$ \\
\hline 80 & 501,5 a & Carioca & 2,18 a & $2,80 \mathrm{a}$ \\
\hline 40 & $483,8 \mathrm{~b}$ & Rosinha & $2,25 \mathrm{a}$ & $4,83 \mathrm{c}$ \\
\hline 16 & $482,9 \mathrm{~b}$ & Rosinha & $2,08 \mathrm{a}$ & $3,72 \mathrm{~b}$ \\
\hline 37 & 515,3 a & Rosinha & $2,24 \mathrm{a}$ & 2,17 a \\
\hline $69 \mathrm{C}$ & 507,2 a & Carioca & $1,90 \mathrm{a}$ & 3,02 a \\
\hline $7 \mathrm{R}$ & $452,1 \mathrm{~b}$ & Rosinha & $2,16 \mathrm{a}$ & $4,24 \mathrm{~b}$ \\
\hline 66 & $458,0 \mathrm{~b}$ & Rosinha & $1,98 \mathrm{a}$ & $2,90 \mathrm{a}$ \\
\hline 58 & 528,6 a & Carioca & 2,33 a & $2,88 \mathrm{a}$ \\
\hline 12 & 518,6 a & Rosinha & $2,48 \mathrm{a}$ & $4,70 \mathrm{c}$ \\
\hline 49 & $463,8 \mathrm{~b}$ & Rosinha & $2,26 \mathrm{a}$ & $4,22 \mathrm{~b}$ \\
\hline 9 & $455,9 \mathrm{~b}$ & Rosinha & $2,25 \mathrm{a}$ & $4,05 \mathrm{~b}$ \\
\hline 1 & 524,0 a & Carioca & $2,11 \mathrm{a}$ & $3,94 \mathrm{~b}$ \\
\hline 3 & 509,5 a & Rosinha & 2,16 a & 3,02 a \\
\hline 41 & $475,9 \mathrm{~b}$ & Carioca & $2,39 \mathrm{a}$ & $2,42 \mathrm{a}$ \\
\hline 27 & 524,9 a & Carioca & $2,23 \mathrm{a}$ & $2,61 \mathrm{a}$ \\
\hline 'rosinha' & $480,0 \mathrm{~b}$ & Rosinha & $2,10 \mathrm{a}$ & $5,27 \mathrm{c}$ \\
\hline ESAL 693 & $416,2 \mathrm{~b}$ & Carioca & $3,48 \mathrm{~b}$ & $2,13 \mathrm{a}$ \\
\hline Média & 496,4 & & 2,14 & 3,43 \\
\hline
\end{tabular}

${ }^{1}$ Notas de 1 (mais aceito pelo consumidor) a 5 (menos aceito pelo consumidor) para tipo de grãos; 1 (mais resistente) a 9 (mais suscetível) para reação a Phaeoisariopsis griseola.

${ }^{2}$ Médias seguidas de mesma letra pertencem ao mesmo grupo, segundo o teste de Scott \& Knott ao nível de $5 \%$ de probabilidade.

Ciênc. agrotec., Lavras, v. 30, n. 1, p. 67-71, jan./fev., 2006 


\section{CONCLUSÃO}

Foram selecionadas famílias de feijoeiro comum superiores em relação a produção e tipo de grãos e resistência à mancha angular, havendo a possibilidade de seleção de linhagens com potencial para ser uma nova cultivar.

\section{AGRADECIMENTOS}

À FAPEMIG pelo apoio financeiro e ao CNPq pela bolsa de iniciação científica.

\section{REFERÊNCIAS BIBLIOGRÁFICAS}

BALDONI, A. B.; SANTOS, J. B. dos. Capacidade de cozimento de grãos de famílias de feijão do cruzamento ESAL 693 x 'rosinha'. Acta Scientiarum, Maringá, 2005. No prelo.

MARQUES JÚNIOR, O. G. M. Eficiência de experimentos com a cultura do feijão. 1997. $80 \mathrm{f}$. Tese (Doutorado em Genética e Melhoramento de Plantas) - Universidade Federal de Lavras, Lavras, 1997.

MENDONÇA, H. A. de; SANTOS, J. B. dos; RAMALHO, M. A. P. Genetic control of common bean reaction to Phaeoisriopsis griseola. Crop Breeding and Applied Biotechnology, Londrina, v. 3, n. 3, p. 209-216, 2003.

NIETSCHE, S.; BORÉM, A.; CARVALHO, G. A.; PAULA JÚNIOR, T. J.; FERREIRA, C. F.; BARROS, E. G. de; MOREIRA, M. A. Genetic diversity of $P$. griseola in the State of Minas Gerais, Brazil. Euphytica, Wageningen, v. 117, p. 77-84, 2001.

PAULA JÚNIOR, T. J. de; ZAMBOLIM, L. Doenças. In: VIEIRA, C.; PAULA JÚNIOR, T. J. de; BORÉM, A. Feijão: aspectos gerais e cultura no estado de Minas Gerais. Viçosa: UFV, 1998. p. 375-433.

PEREIRA, H. S.; SANTOS, J. B. dos; ABREU, A. de F. B. Seleção de linhagens arbustivas de feijoeiro com grãos tipo 'carioca', resistência a antracnose e mancha angular e alta produtividade. Pesquisa Agropecuária Brasileira, Brasília, v. 39, n. 3, p. 209-215, 2004.
POMPEU, A. S. Feijão. In: FURLANI, A. M. C.; VIÉGAS, G. P. O melhoramento de plantas no Instituto Agronômico. Campinas: IAC, 1993.

RAMALHO, M. A. P.; ABREU, A. de F. B. Cultivares. In: VIEIRA, C.; PAULA JÚNIOR, T. J. de; BORÉM, A. Feijão: aspectos gerais e cultura no estado de Minas Gerais. Viçosa: UFV, 1998. p. 435-449.

RAMALHO, M. A. P.; SANTOS, J. B.; ZIMMERMANN, M. J. de O. Genética quantitativa em plantas autógamas: aplicação ao melhoramento do feijoeiro. Goiânia: UFG, 1993. $271 \mathrm{p}$.

RAVA, C. A.; PURCHIO, A. F.; SARTORATO, A. Caracterização de patótipos de Colletotrichum lindemuthianum que ocorrem em algumas regiões produtoras de feijoeiro comum. Fitopatologia Brasileira, Fortaleza, v. 19, p. 167-173, 1994.

SARTORATO, A. Resistência do feijoeiro comum à mancha angular. In: CONGRESSO BRASILEIRO DE MELHORAMENTO DE PLANTAS, 1., 2001, Goiânia, GO. Resumos... Goiânia: SBMP/EMBRAPA, 2001. CD-ROM.

SARTORATO, A.; RAVA, C. A. Principais doenças do feijoeiro comum e seu controle. Brasília: EMBRAPA, 1994. $300 \mathrm{p}$.

SILVA, M. V. da; SANTOS, J. B. dos. Identificação de marcador RAPD ligado ao alelo $C o .4^{2}$ de resistência do feijoeiro comum ao agente causal da antracnose. Ciência e Agrotecnologia, Lavras, v. 25, n. 5, p. 1097-1104, 2001.

VENCOVSKY, R.; BARRIGA, P. Genética biométrica aplicada ao fitomelhoramento. Ribeirão Preto: Sociedade Brasileira de Genética, 1992. 496 p.

VIEIRA, F. C. Controle genético da reação do feijoeiro ao Phaseoisariopsis griseola e seleção de famílias baseada em caracteres agronômicos. 2004. 31 p. Dissertação (Mestrado em Genética e Melhoramento de Plantas) Universidade Federal de Lavras, Lavras, 2004. 